\title{
Effectiveness of Paradox Therapy on COVID-19 Anxiety in Adult Population in Jahrom
}

\author{
Razieh Eatesamipour (iD ${ }^{1,{ }^{*}}$, Khadigeh Ramazanzade Moghadam (iD) ${ }^{1}$ and Borzoo Amirpour (iD) ${ }^{2}$ \\ ${ }^{1}$ Department of Psychology, Masters of Clinical Psychology, Payame Noor University, Tehran, Iran \\ ${ }^{2}$ Department of Psychology, Faculty of Psychology, Payame Noor University, Tehran, Iran \\ "Corresponding author: Department of Psychology, Faculty of Psychology, Payame Noor University, Tehran, Iran. Email: r.eatesami@gmail.com \\ Received 2021 October 25; Accepted 2021 November 30.
}

\begin{abstract}
Background: Since COVID-19 is a new disease with devastating effects worldwide, its emergence and spread aroused confusion, anxiety, and fear among the public.

Objectives: The present study aimed to evaluate the effectiveness of paradox therapy on the COVID-19 anxiety in adult population in Jahrom, Iran.

Methods: This research was a quasi-experimental study with a pretest-posttest design and a two-month follow-up. The research population encompassed all individuals willing to participate in the present study who were residing in Jahrom during 2020-2021. According to the COVID-19 Anxiety Scale, 30 participants with high levels of COVID-19 anxiety were selected using the convenience sampling method and were randomly assigned to experimental $(n=15)$ and control $(n=15)$ groups. The experimental group received eight sessions of paradox therapy; however, the control group remained on the waiting list. The research tools included structured clinical interviews and the COVID-19 Anxiety Scale. Finally, the collected data were analyzed using Friedman's statistical method.

Results: The results showed that paradox therapy was effective in decreasing COVID-19 anxiety $(\mathrm{P}=0.001)$ and its psychological ( $\mathrm{P}$ $=0.001)$ and physical $(\mathrm{P}=0.002)$ symptoms, and the scores of the COVID-19-induced psychological and physical symptoms in the posttest phase were significantly lower in the intervention group than the control group $(\mathrm{P}<0.001)$. The decrease was noticed in the follow-up phase as well.

Conclusions: According to the results, paradox therapy can effectively reduce anxiety and its psychological and physical symptoms due to COVID-19.
\end{abstract}

Keywords: Paradox Therapy, Anxiety, COVID-19 Disease, Adult Population

\section{Background}

The COVID-19 pandemic has had a profound effect on individuals' mental health worldwide as it has increased the prevalence of anxiety and depression (1). The prevalence of mental disorders and sleep problems is several times higher during the COVID-19 pandemic compared to the previously reported statistics (2). Numerous crosssectional studies worldwide have confirmed the negative impact of COVID-19 on individuals' mental health. However, few studies have examined the risk factors for the pandemic and their impacts on mental health (3).

Since the trend and prognosis of this disease are unknown in different individuals, and there is not enough information about the nature of the disease, similar to other life-threatening diseases decreasing life expectancy, it may have significant psychological side-effects such as fear of death, anxiety, depression, and stress on the infected indi- viduals and influence the course of recovery (4). According to Lai et al. (5), depression, anxiety, insomnia, and distress are psychological issues involved in the COVID-19 disease. Qiu et al. (6) found out that mental health problems such as panic, anxiety, depression, fear, denial, and despair are the most fundamental traumatic psychological reactions in most affected individuals and those exposed to the disease outbreak. In general, a disease epidemic can significantly lead to increased worry and anxiety and even severe mental illnesses in individuals (7). Although researchers have spared their efforts to compete for the production of the COVID-19 vaccine, since this does not work fast enough to control the epidemic, mass communication restrictions (extensive restrictions) and infection prevention strategies (e.g., travel restrictions, quarantine, and self-quarantine) have been adopted to prevent the spread of the virus (8).

In general, the spread of infectious diseases can lead

Copyright (c) 2021, Modern Care Journal. This is an open-access article distributed under the terms of the Creative Commons Attribution-NonCommercial 4.0 International License (http://creativecommons.org/licenses/by-nc/4.0/) which permits copy and redistribute the material just in noncommercial usages, provided the original work is properly cited. 
to increased anxiety, and this anxiety, in turn, has unpleasant effects on individuals' mental states (9). Anxiety is one of the most common mental disorders, leading to a widespread, unpleasant, and ambiguous feeling of fear and stress of unknown origin and physiological arousal (10). The COVID-19-induced anxiety stems from infection by the coronavirus, mainly because it is unknown and creates cognitive ambiguity (11). Due to the novelty of the COVID-19 disease, little research has addressed the disease anxiety and its treatment options. Previous research on treatments using psychotherapeutic approaches have indicated that they were more effective in treating depressive symptoms than anxiety. The most common and practical strategies were exercise, concentration, and breathing exercises. Using mindfulness and finding it helpful were also associated with lower depression and anxiety symptoms. No other strategy was associated with improved mental health. Individuals' prior knowledge of psychotherapy strategies may play a role in mental health management in unprecedented public health events such as the global pandemic. The widespread promotion of these techniques in the community to manage general distress in such situations may also contribute (12). The simultaneous provision of treatment and psychological support may reduce the burden of mental illnesses and improve individuals' health.

This article used the paradoxical psychotherapy (PT) model to treat COVID-19 anxiety. The PT model, called "Paradox + Timetable $=$ Cure (PTC)," as a treatment method for psychological disorders, has overcome many limitations and shortcomings of other methods. The PTC treatment is a systemic-behavioral model with two fundamental components. The first component, "paradox," refers to prescribing a behavioral symptom or disorder. According to this model, clients must reconstruct behavioral and disorder symptoms. In other words, they must reconstruct the same behaviors and symptoms they suffer. The second component, "timetable," is a task, according to which the client is supposed to reconstruct and experience the same symptom or behavior prescribed in the form of paradox at a specific time for a certain period. The combinations of these two therapeutic techniques forms a paradoxical timetable. The paradoxical timetable creates therapeutic changes based on four mechanisms. The first mechanism is grammaticality or artificiality, in which an individual activates and experiences symptoms when he/she desires.

The second mechanism is formed when a patient experiences artificial symptoms as such, it is the disconnection of the relationship between symptoms and anxiety. When the disorder symptoms are artificially reconstructed and experienced, the individual is exposed to a new phenomenon and experience. Symptoms may still be present; however, they contain no bitter taste of anxiety. When a behavior or a symptom is without anxiety, it will no longer be pathogenic as such the relationship between symptoms and anxiety is disconnected. The underlying principle of this treatment is to eliminate anxiety. The third mechanism is to change the symptom's meaning. When a client reconstructs his/her symptom, the symptoms' meanings change for him/her and his/her surrounding system (i.e., family members) as such the client is supposed to experience them voluntarily. The fourth mechanism is the "ego" strength. According to the psychoanalytic model, when the relationship between symptoms and anxiety is disconnected, the "ego" can become powerful once more. The strength and weakness of the "ego," which differ in each person and are functions of their past experiences and individual differences, are considered the main factors affecting the speed and slowness of realizing such power (13). This method has been effective in treating a large number of psychological disorders (American Psychiatric Association (2013), including a wide range of anxiety disorders, obsessive-compulsive disorder and relevant disorders, trauma- and stress-related disorders, and physical symptom disorders (13). Accordingly, given the effectiveness of this treatment model in treating anxiety disorders, its role in decreasing the level of COVID-19 anxiety may be significant. The present study was to detect whether paradox therapy can be used as an effective psychotherapy method to reduce and treat anxiety in patients with COVID19 anxiety.

\section{Methods}

This study aimed to evaluate the effectiveness of paradox therapy in treating COVID-19 anxiety. This quasiexperimental research design used pretest-posttest with a control group and follow-up. The research population encompassed all individuals willing to participate in the present study who were residing in Jahrom during 2020 - 2021. To this end, a call for participation in an intervention study on COVID-19 anxiety was first published via social networks, and the applicants were assessed using the COVID-19 anxiety questionnaire. Then 30 participants with high levels of COVID-19 anxiety were selected using the convenience sampling method and were randomly assigned to experimental $(n=15)$ and control $(n=15)$ groups. Considering Cohen's table with an effect size of 0.5 and a test power of 0.75 , the sample size was estimated to be15 persons per group. In the preliminary assessment, the partic- 
ipants received some explanations on the research design, and they were examined regarding inclusion criteria. The inclusion criteria were being aged above 20 years, holding a diploma and having higher education, being motivated and satisfied to take part in intervention sessions, and not suffering from comorbid psychological disorders associated with COVID-19 anxiety. As presented in Table 1, the experimental group received eight sessions of paradox therapy intervention, and the control group remained on the waiting list. Due to the following readons Friedman's non-parametric test was used to examine the two groups: The significance level of the Kolmogorov-Smirnov test (the data normality prerequisite) in the two groups regarding the difference between the posttest of physical anxiety $(\mathrm{P}=$ 0.001) and the follow-up physical symptoms of the COVID19 anxiety $(\mathrm{P}=0.001)$ was $\mathrm{P}<0.05$; no normal distribution of the collected data; the significance level of the Leven test (non-observance of homogeneity of variances) in the follow-up phase of COVID-19 anxiety $(\mathrm{P}=0.018)$ and its psychological symptoms $(\mathrm{P}=0.034)$ and the follow-up physical symptoms of the COVID-19 anxiety $(\mathrm{P}=0.026)$; and the non-establishment of regression slope default in the COVID-19 anxiety index and its physical symptoms. Friedman's statistical test was used to evaluate the effectiveness of paradox therapy in reducing the COVID-19 anxiety and its physical and psychological symptoms. SPSS software version 21 was used in this study to analyze the collected data. This study was extracted from a master's thesis approved by the Ethics Committee in Biomedical Research at the Payame Noor University (Code: IR.PNU.REC.1400.085).

\subsection{Data Collection Tools}

\subsubsection{COVID-19 Anxiety Scale}

This questionnaire was developed by Alipour et al. to determine the level of COVID-19 anxiety in a clinical sample. It consists of 18 items and two psychological and physical factors and provides a total score (11). Items 1-9 address the psychological subscale, and Items 10-18 deal with the physical subscale. The scale is scored based on a four-point Likert scale ranging from 0 to 3 . Moreover, the minimum and maximum score of this questionnaire are 0 and 54 , respectively, with scores 0 - 18 indicating mild anxiety, 19 - 36 indicating moderate anxiety, and 37 - 54 indicating severe anxiety requiring intervention. The scores of psychological and physical symptoms ranged from 0 to 27, depending on the number of items per factor. Cronbach's alpha coefficients determined the reliability of the first factor (a $=0.879)$, the second factor $(\mathrm{a}=0.861)$, and the total questionnaire $(\mathrm{a}=0.919)$. To evaluate the criterion validity of this questionnaire, its correlation of this tool with the General Health Questionnaire-28 (GHQ-28) was estimated. The results showed that the reliability of the COVID-19 Anxiety Scale with the total score of the GHQ-28 and the components of anxiety, physical symptoms, social dysfunction, and depression were $0.483,0.507,0.418,0.333$, and 0.269 , respectively. In this regard, the coefficients were significant at $\mathrm{P}=0.01$ (11).

\subsubsection{Intervention}

This protocol was performed in the experimental group in 8 two-hour sessions once a week. The pretest was performed before implementing the protocol, and the posttest was performed after the full implementation of the treatment protocol. The follow-up test was run two months later to evaluate the treatment stability.

\section{Results}

The experimental and control groups' age mean and standard deviation were $27.60 \pm 8.83$ and $31.07 \pm 9.28$, respectively. The chi-square test revealed no significant difference between the experimental and control groups in terms of gender $(\mathrm{P}=1.00)$, marital status $(\mathrm{P}=0.713)$, employment status $(\mathrm{P}=0.116)$, and level of education $(\mathrm{P}=0.079)$. Table 2 presents the other demographic features of the participants. Table 3 shows the effectiveness of PTC in the experimental group in the posttest and follow-up stages and reduced COVID-19 anxiety $(\mathrm{P}=0.001)$. In Table 4, PTC was effective in the experimental group in the posttest and follow-up phases $(\mathrm{P}=0.001)$ and reduced physical symptoms caused by the COVID-19 anxiety. As presented in Table 5, PTC was effective in the experimental group in the posttest and follow-up phases and reduced psychological symptoms caused by the COVID-19 anxiety $(\mathrm{P}=0.001)$.

\section{Discussion}

The findings of this study showed that paradox therapy effectively reduced the COVID-19 anxiety in individuals suffering from this type of anxiety. According to Table 3, the PTC treatment was effective in the experimental group in the posttest and follow-up phases and reduced the COVID19 anxiety $(\mathrm{P}=0.001)$. This finding implies the effectiveness of PTC in reducing anxiety in individuals with this type of anxiety. The present findings are in line with some other studies (13-17) regarding the effectiveness of paradox therapy in reducing the physical symptoms of anxiety.

Some studies examining the effectiveness of PTC in reducing anxiety symptoms compared to pharmacotherapy 


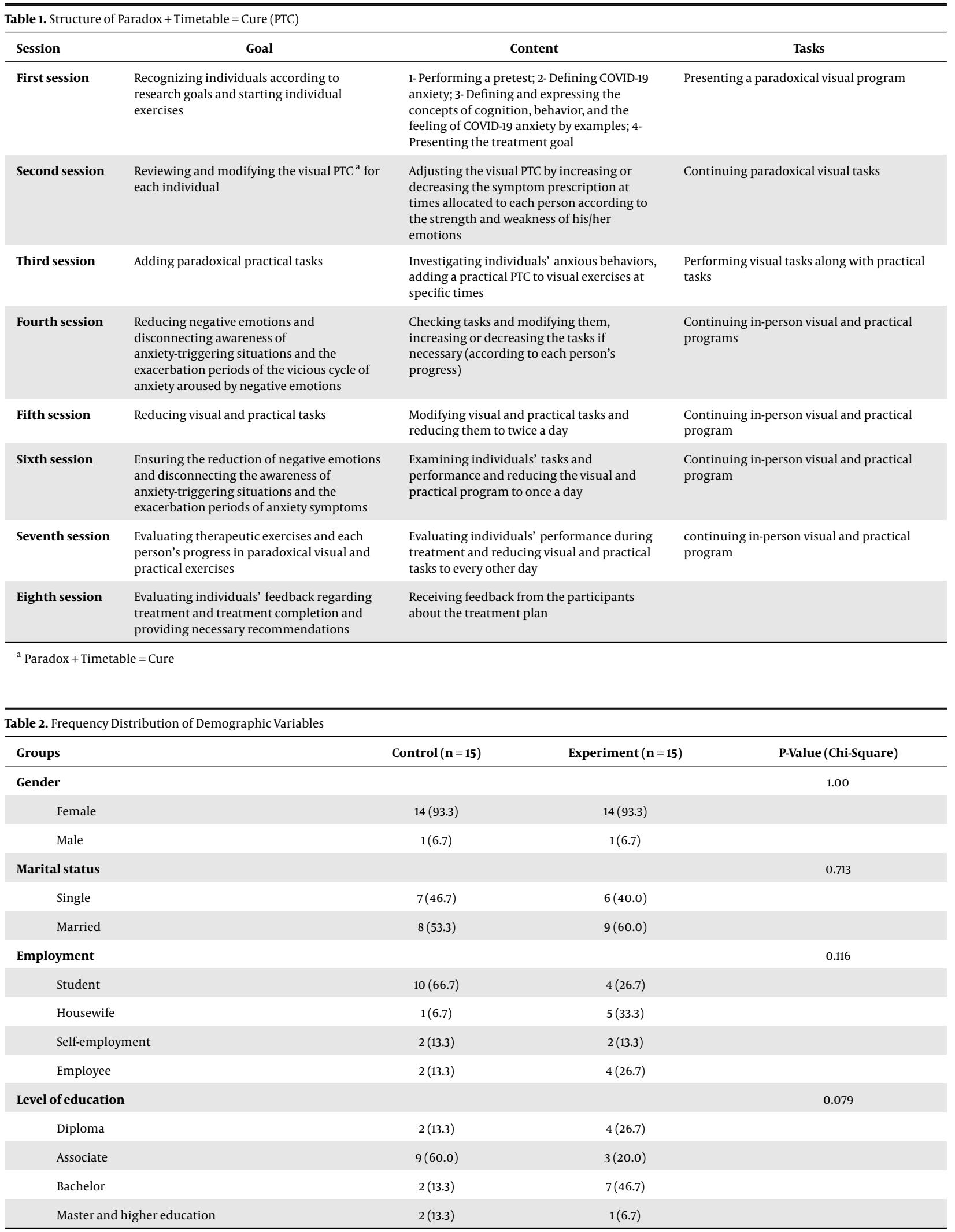




\begin{tabular}{|c|c|c|c|c|}
\hline \multirow{2}{*}{ Group } & \multicolumn{3}{|c|}{ Time } & \multirow{2}{*}{ Friedman's Test } \\
\hline & Before Median (Q1 - Q3) & After Median (Q1 - Q3) & $\begin{array}{l}\text { Two-month follow-up Median } \\
\text { (Q1 - Q3) }\end{array}$ & \\
\hline Experimental & $28(21-32)$ & $3(1-8)$ & $2(0-7)$ & 0.001 \\
\hline Control & $25(14-32)$ & $14(9-18)$ & $14(6-18)$ & 0.796 \\
\hline Mann-Whitney U test & 0.430 & 0.001 & 0.001 & \\
\hline \multirow{2}{*}{ Group } & & Time & & \multirow{2}{*}{ Friedman's Test } \\
\hline & Before Median (Q1 - Q3) & After Median (Q1 - Q3) & $\begin{array}{l}\text { Two-month follow-up Median } \\
\text { (Q1 - Q3) }\end{array}$ & \\
\hline Control & $7(1-13)$ & $4(2-4)$ & $2(1-4)$ & 0.285 \\
\hline Mann-Whitney U test & 0.189 & 0.001 & 0.002 & \\
\hline \multirow{2}{*}{ Group } & \multicolumn{3}{|c|}{ Time } & \multirow{2}{*}{ Friedman's Test } \\
\hline & Before Median (Q1 - Q3) & After Median (Q1 - Q3) & $\begin{array}{l}\text { Two-month follow-up Median } \\
\text { (Q1 - Q3) }\end{array}$ & \\
\hline Experimental & $18(15-23)$ & $3(1-7)$ & $2(0-7)$ & 0.001 \\
\hline Control & $18(12-22)$ & $11(9-14)$ & $10(5-14)$ & 0.439 \\
\hline Mann-Whitney U test & 0.708 & 0.001 & 0.001 & \\
\hline
\end{tabular}

and cognitive-behavioral therapy indicate that the clinical significance and improvement percentage of the PTC treatment are higher than other therapies and that the PTC treatment is an appropriate and quick way to reduce anxiety symptoms $(14,16,18)$. In their research, Besharat and Naghipour (14) examined the effect of the PTC model on an individual with anxiety disorder. Their study showed the effectiveness of this psychotherapy model and its acceptable stability after a 28-month follow-up period. In another study, Besharat (18) also examined the effect of the PTC model on an individual with social anxiety disorder. The results of the three-session treatment and the threeyears follow-up period indicated satisfactory changes as well as the stability and continuity of the treatment. In general, studies have confirmed the PTC model as a practical, short-term, and economical method in treating psychological disorders, including anxiety disorders, with the slightest likelihood of disease recurrence in mental disorders (13).

The PTC model was used due to its shorter treatment duration compared to long-term treatments making individuals abandon it half-finished. This model plays a critical role in treating individuals highly resistant to treatment as it is based on symptom prescription. In this model, the therapist's instructions and prescribed tasks are all functions of the principles minimizing the anxiety of performing tasks and maximizing the patient's potentials to obey. The model principles, including inseparability of paradox from PTC, prescription of the same disease symptoms, the delayed execution of tasks, and the exclusive reliance on practical treatment techniques, pave the way for a practical, emotional experience and consequently the realization of therapeutic changes as quickly and efficiently as possible. The ultimate goal of this fast and highly shortterm treatment is to make some changes in the "ego" level and in its relation and distance to the "id" and the "superego," thereby leading to the "ego" strength (i.e., a degree of strength predicting the stability of treatment changes and the sharp decline in the recurrence of symptoms) (19).

According to the findings, paradox therapy effectively reduces the physical symptoms of COVID-19 anxiety in individuals suffering from this type of anxiety. The findings in Table 4 indicate that the PTC treatment was effective in the experimental group in the posttest and follow-up phases ( $P$ $=0.002$ ) and reduced the physical symptoms of the COVID19 anxiety. This concept indicates the effectiveness of PTC in 
reducing the physical symptoms of COVID-19 anxiety. The present findings are in line with some other studies (13-17) regarding the effectiveness of paradox therapy in reducing the physical symptoms of anxiety.

Coronavirus research has indicated that anxious individuals afraid of COVID-19 experience a coherent set of unpleasant and physiological symptoms aroused by thoughts or information about this infectious disease (20, 21).

Excess COVID-19 anxiety may be a unique type of health anxiety, one of the unique symptoms of which is the fear of infection by COVID-19. According to previous studies on the COVID-19, anxiety, disgust, intolerance of hesitation, anxiety sensitivity, and fear of physical arousal are the predictors of excess anxiety triggered by COVID-19 $(22,23)$.

One type of anxiety is disease-related anxiety. In particular, infectious diseases often trigger extreme fear and anxiety, thereby leading to many widespread disturbances in behavior and psychological well-being in society (24).

The PTC model is one of the effective models in treating psychological disorders such as anxiety $(14,16,18)$. The model had remarkable features in terms of its therapeutic goals. The elimination of symptoms and coercive and imposed behaviors in individuals significantly contribute to the concerned treatment. The PTC model has been used to eliminate such symptoms. In this technique, individuals voluntarily face some of their symptoms (i.e., physical symptoms associated with the COVID-19 anxiety, including palpitations, headaches, tremors, fatigue and lethargy, and nightmares) and utilize complete reconstruction to make the symptoms and the experiences of those compulsive symptoms totally optional. Accordingly, any optional symptom or behavior is no longer a disease, a problem, or a problematic issue.

According to the results, paradox therapy effectively reduces the psychological symptoms of COVID-19 anxiety in individuals suffering from this type of anxiety. Table 5 indicates that the PTC treatment in the experimental group was effective in the posttest and follow-up phases and reduced the psychological symptoms of the COVID-19 anxiety $(\mathrm{P}=0.001)$. This finding indicates the effectiveness of the PTC treatment in reducing the psychological symptoms of the COVID-19 anxiety. The present findings are consistent with those in previous studies on the effectiveness of paradox therapy in reducing psychological symptoms of anxiety $(14,15,17,18)$.

Psychological factors play a vital role in how individuals deal with the threat of an epidemic infection and its consequences (e.g., the loss of a loved one). Although many individuals cope well with the threat of an epidemic, many others experience high levels of anxiety or further mental health problems such as anxiety disorders and other clinical conditions (25).

The main problem with anxious individuals is that they fill their minds with worrying illusions, thereby increasing their anxiety. Anxiety minimizes or sometimes completely destroys an individual's power (26) and affects his/her psychological well-being. Moreover, lack of scientific information also exacerbates such anxiety. In some cases, individuals look for more information to relieve their anxiety. Anxiety can make individuals misdiagnose true and false information as such, they may be exposed to false news (11), thereby increasing the level of their anxiety induced by a new infectious disease.

The presence of disease anxiety in the community, especially in families at risk of disease (due to living with a sick person or a person having more contact with COVID19 patients), indicates the need to use a therapeutic intervention. As an alternative treatment to prevent responses, PTC can eliminate maladaptive thinking strategies about anxiety. Paradox therapy is one of Frankel's semantic therapy methods, which is used to help an individual realize that he/she does not look like his/her perceptual symptoms. Paradoxically, thoughts and feelings are typically designed to over-stimulate the way of thinking about fear or unwanted behavior (27).

One of the goals of the PTC method was to eliminate all negative emotions arising from anxiety and its psychological symptoms. In the paradox therapy exercises, individuals are asked not to control their thoughts, including those about one's death and their loved ones, fear of hospitalization, fear of infection with COVID-19 as well as relevant practices such as hand washing and disinfection of one's hands and stuff. This is because one of the therapeutic goals is to communicate with thoughts to prevent resistance or complex perceptual analysis to eliminate maladaptive thinking strategies about being worry and having inflexible control over threats (13). This awareness arouses when the psychological pressures imposed by controlling and blaming thoughts associated with washing and disinfecting hands and equipment and negative feelings about illness and death are eliminated. This implies that when the psychological stress of negative emotions is high, individuals having difficulty in regulating their emotions are inclined to rumination and anger posed by the condition to moderate their mental energy. By relieving COVID19 anxiety and disconnecting thoughts and their behavioral cycles, this type of therapy makes individuals regulate their emotions better by reinforcing their "ego" and promoting awareness. 


\subsection{Limitations}

Regarding the limitations of the present study, several points need to be mentioned. Diagnosing COVID-19 anxiety was exclusively limited to a questionnaire as such this study can also encompass the limitations mentioned for the questionnaires. This research was conducted in Jahrom, and necessary precautions should be taken in generalizing the findings. The lack of research on physical and psychological indicators in COVID-19 patients, small sample size, and the impossibility of holding in-person meetings were the other limitations of the present study.

\subsection{Conclusions}

According to the findings, paradox therapy can be used as an appropriate model to treat COVID-19 anxiety and its physical and psychological symptoms in patients.

\section{Acknowledgments}

The authors would like to express their gratitude to all participants and those who contributed to this research.

\section{Footnotes}

Authors' Contribution: RE acted as the principal investigator of the current study, co-designed the study, supervised the procedure for data collection and quality control, and helped in writing the paper. K RM acted as data collection, and also contributed to analyzing the data and writing the paper. BA helped interpreting the data, and writing the paper. Provided his appreciated input for data interpretation and writing the paper. RE, KRM. BA gave their valued impact for data collection and data quality control. All authors have read, edited, and approved the final version of the manuscript.

Conflict of Interests: The authors declare that there is no conflict of interest.

Ethical Approval: This study was approved by the Ethics Committee in the Biomedical Research Department of Payam-e-Noor University (Code: IR.PNU.REC.1400.085).

Funding/Support: The author(s) received no specific funding for this research.

Informed Consent: Informed written consent was obtained from the participants.

\section{References}

1. Egan SJ, McEvoy P, Wade TD, Ure S, Johnson AR, Gill C, et al. Unguided low intensity cognitive behaviour therapy for anxiety and depression during the COVID-19 pandemic: A randomised trial. Behav Res Ther. 2021;144:103902. doi: 10.1016/j.brat.2021.103902. [PubMed: 34174706]. [PubMed Central: PMC8197681].
2. Olagunju AT, Bioku AA, Olagunju TO, Sarimiye FO, Onwuameze OE, Halbreich U. Psychological distress and sleep problems in healthcare workers in a developing context during COVID-19 pandemic: Implications for workplace wellbeing. Prog Neuropsychopharmacol Biol Psychiatry. 2021;110:110292. doi:10.1016/j.pnpbp.2021.110292. [PubMed: 33662533]. [PubMed Central: PMC7920822].

3. Turna J, Zhang J, Lamberti N, Patterson B, Simpson W, Francisco AP, et al. Anxiety, depression and stress during the COVID-19 pandemic: Results from a cross-sectional survey.J Psychiatr Res. 2021;137:96-103. doi: 10.1016/j.jpsychires.2021.02.059. [PubMed: 33667763]. [PubMed Central: PMC8548032].

4. Zhu N, Zhang D, Wang W, Li X, Yang B, Song J, et al. A Novel Coronavirus from Patients with Pneumonia in China, 2019. N Engl J Med. 2020;382(8):727-33. doi: 10.1056/NEJMoa2001017. [PubMed: 31978945]. [PubMed Central: PMC7092803].

5. Lai J, Ma S, Wang Y, Cai Z, Hu J, Wei N, et al. Factors Associated With Mental Health Outcomes Among Health Care Workers Exposed to Coronavirus Disease 2019. JAMA Netw Open. 2020;3(3). e203976. doi: 10.1001/jamanetworkopen.2020.3976. [PubMed: 32202646]. [PubMed Central: PMC7090843].

6. Qiu J, Shen B, Zhao M, Wang Z, Xie B, Xu Y. A nationwide survey of psychological distress among Chinese people in the COVID-19 epidemic: implications and policy recommendations. Gen Psychiatr. 2020;33(2). e100213. doi: 10.1136/gpsych-2020-100213. [PubMed: 32215365]. [PubMed Central: PMC7061893].

7. Roy D, Tripathy S, Kar SK, Sharma N, Verma SK, Kaushal V. Study of knowledge, attitude, anxiety \& perceived mental healthcare need in Indian population during COVID-19 pandemic. Asian J Psychiatr. 2020;51:102083. doi: 10.1016/j.ajp.2020.102083. [PubMed: 32283510]. [PubMed Central: PMC7139237].

8. World Health O. Mental health and psychosocial considerations during the COVID-19 outbreak, 18 March 2020. Geneva: World Health Organization; 2020. Report No.: WHO/2019-nCoV/MentalHealth/2020.1.

9. Taylor S, Landry CA, Paluszek MM, Fergus TA, McKay D, Asmundson GJG. Development and initial validation of the COVID Stress Scales. J Anxiety Disord. 2020;72:102232. doi: 10.1016/j.janxdis.2020.102232. [PubMed: 32408047]. [PubMed Central: PMC7198206].

10. Asnaani A, Tyler J, McCann J, Brown L, Zang Y. Anxiety sensitivity and emotion regulation as mechanisms of successful CBT outcome for anxiety-related disorders in a naturalistic treatment setting. J Affect Disord. 2020;267:86-95. doi: 10.1016/j.jad.2020.01.160. [PubMed: 32063577].

11. Alipour A, Ghadami A, Alipour Z, Abdollahzadeh H. [Preliminary validation of the Corona Disease Anxiety Scale (CDAS) in the Iranian sample]. $J$ Health Psychol. 2020;8(32):163-75. Persian. doi: 10.30473/HPJ.2020.52023.4756.

12. Gulliver A, Banfield M, Batterham PJ, Calear AL, Farrer LM, Dawel A, et al. Effects of previous exposure to psychotherapeutic strategies on depression and anxiety symptoms during the COVID-19 pandemic. BJPsych Open. 2021;7(1). e38. doi: 10.1192/bjo.2020.170. [PubMed: 33461641]. [PubMed Central: PMC7844171].

13. Besharat MA. Paradox + Timetable = Cure (PTC): Perfect model of treatment for psychological disorders (A practical guide). Tehran: Roshd Press; 2017. Persian.

14. Besharat MA, Naghipoor M. [The Application of a New Model of Paradox Therapy for the Treatment of Illness Anxiety Disorder: A Case Report]. Clin Case Rep Int. 2019;3:1100. Persian. doi: 10.26226/morressier.5d1a037757558b317a140728. 
15. Besharat M A, M N. [Paradox therapy for the treatment of social anxiety disorder: A case study]. J Syst Integr Neurosci. 2020;6(1):5. Persian. doi: $10.15761 /$ jsin.1000213.

16. Besharat M. [The application of paradox therapy for the treatment of body dysmorphic disorder: A case report]. J Psychol Sci. 2021;19(95):1371-87. Persian.

17. Wardani LK, Melda B, Kurnia D, Azizah N. The Effect of Paradoxical Intension Session 1 Logotheraphy on Prisoners Anxiety in LAPAS Kediri. J Ners. 2020;15(2):452-6.

18. Besharat MA. [Evaluating the effectiveness of paradox therapy for the treatment of social anxiety disorder: A case study].J psychol Science. 2019;18(76):383-96. Persian.

19. Besharat MA. [Evaluating the effectiveness of paradox therapy for the treatment of obsessive-compulsive disorder: A case study]. J Psychol Stud. 2019;14(4):7-36. Persian. doi: 10.22051/PSY.2019.23631.1801.

20. Evren C, Evren B, Dalbudak E, Topcu M, Kutlu N. Measuring anxiety related to COVID-19: A Turkish validation study of the Coronavirus Anxiety Scale. Death Stud. 2020:1-7. doi: 10.1080/07481187.2020.1774969. [PubMed: 32490730].

21. Lee SA. Coronavirus Anxiety Scale: A brief mental health screener for COVID-19 related anxiety. Death Stud. 2020;44(7):393-401. doi: 10.1080/07481187.2020.1748481. [PubMed: 32299304].

22. Ojalehto HJ, Abramowitz JS, Hellberg SN, Butcher MW, Buch- holz JL. Predicting COVID-19-related anxiety: The role of obsessive-compulsive symptom dimensions, anxiety sensitivity, and body vigilance. J Anxiety Disord. 2021;83:102460. doi 10.1016/j.janxdis.2021.102460. [PubMed: 34352520]. [PubMed Central: PMC8318675].

23. Wheaton MG, Messner GR, Marks JB. Intolerance of uncertainty as a factor linking obsessive-compulsive symptoms, health anxiety and concerns about the spread of the novel coronavirus (COVID-19) in the United States. J Obsessive Compuls Relat Disord. 2021;28:100605. doi: 10.1016/j.jocrd.2020.100605. [PubMed: 33251098]. [PubMed Central: PMC7681070].

24. Yao $\mathrm{H}$, Chen JH, Xu YF. Patients with mental health disorders in the COVID-19 epidemic. Lancet Psychiatry. 2020;7(4). e21. doi 10.1016/S2215-0366(20)30090-0. [PubMed: 32199510]. [PubMed Central: PMC7269717].

25. Taylor S. The psychology of pandemics: Preparing for the next global out break of infectious disease. Cambridge Scholars Publishing; 2019.

26. Rubin GJ, Wessely S. The psychological effects of quarantining a city. BMJ. 2020;368:m313. doi: 10.1136/bmj.m313. [PubMed: 31992552].

27. Lewis MH. Amelioration of obsessive-compulsive disorder using paradoxical intention. Logotherapy and Existential Analysis. Springer, Cham;2016. p. 175-8. 